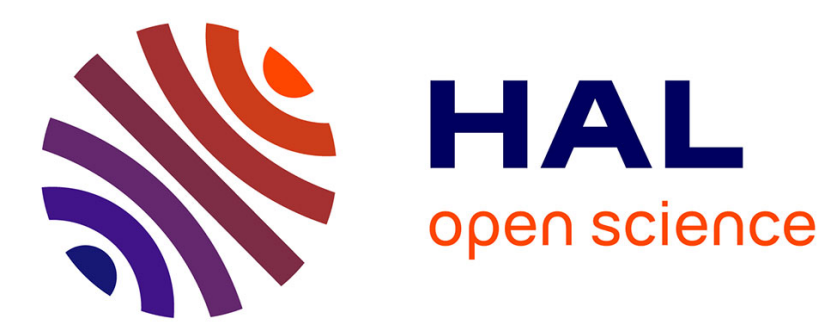

\title{
The wave distribution function in a hot magnetospheric plasma' The direct problem
}

\author{
O Santol, Michel Parrot
}

\section{To cite this version:}

O Santol, Michel Parrot. The wave distribution function in a hot magnetospheric plasma' The direct problem. Journal of Geophysical Research Space Physics, 1996, 10.1029/95JA03510 . insu-03210089

\section{HAL Id: insu-03210089 \\ https://hal-insu.archives-ouvertes.fr/insu-03210089}

Submitted on 27 Apr 2021

HAL is a multi-disciplinary open access archive for the deposit and dissemination of scientific research documents, whether they are published or not. The documents may come from teaching and research institutions in France or abroad, or from public or private research centers.
L'archive ouverte pluridisciplinaire HAL, est destinée au dépôt et à la diffusion de documents scientifiques de niveau recherche, publiés ou non, émanant des établissements d'enseignement et de recherche français ou étrangers, des laboratoires publics ou privés. 


\title{
The wave distribution function in a hot magnetospheric plasma: The direct problem
}

\author{
O. Santolík ${ }^{1}$ and M. Parrot \\ Laboratoire de Physique et Chimie de l'Environnement, Centre National de la Recherche \\ Scientifique, Orléans, France
}

\begin{abstract}
Studying electromagnetic waves in a magnetospheric plasma, it is often important to find their directions of propagation. When the assumption of a plane wave is not valid, the determination of the wave distribution function (WDF) is required. The WDF specifies the distribution of wave-energy density with respect to the frequency and the wave-normal direction. An alternative approach to the estimation of the experimentally accessible data for a given WDF is suggested with the hot-plasma and/or Doppler effects taken into account. The method is based on an explicit frequency dependence of the WDF. Examples of solutions, including the determination of the wave-refractive index, the wave-growth rate, the group velocity, and theoretical predictions of experimental data at predefined frequencies, are given in different cases. First, whistler-mode waves in a hot plasma near the geostationary orbit were studied. Second, the low-frequency waves observed by the low-altitude satellite Freja in the auroral zone were subjected to theoretical analysis. A complex, multimodal structure of the wave characteristics was found, mainly around the resonance angle. In the case of extremely short-wavelength waves, the influence of the Doppler effect produced qualitative changes of the wave-normal dependence of wave parameters.
\end{abstract}

\section{Introduction}

The in situ measurement of fluctuating fields is an important tool for investigating wave phenomena in space plasmas. Usually, several components of the electric and magnetic fields are simultaneously measured, providing information about the wave propagation. The following two general approaches can be chosen: (1) the presence of a single plane wave is supposed or (2) a set of waves with a continuous distribution of wave-normal directions is studied.

The first alternative has been extensively developed by many authors [e.g., Means, 1972; McPherron et al., 1972; Arthur et al., 1976]. The normal direction of a plane wave is determined by various procedures based on the assumption of the perpendicularity of the wave magnetic field to the wave normal. Methods to verify the initial assumption of the single plane wave were also worked out [e.g., McPherron et al., 1972; Samson and Olson, 1980]. Their application to the measured data has shown that, in some cases, the wave field is more complicated. Especially, the data on hiss events are not consistent with the idea of a single plane wave [Par-

\footnotetext{
${ }^{1}$ Now at Faculty of Mathematics and Physics, Charles University, Prague, Czech Republic.

Copyright 1996 by the American Geophysical Union.
}

Paper number 95JA03510.

0148-0227/96/95JA-03510\$09.00 rot and Lefeuvre, 1986]. At a given frequency, several propagation directions can simultaneously be observed and several wave modes may also be involved. In such a case, the subject of analysis must be a general wave continuum.

In order to describe this continuum, the concept of the wave distribution function (WDF) was proposed by Storey and Lefeuvre [1974]. Using an analogy with the particle distribution function from the kinetic theory of gases, the WDF was introduced as a distribution $F_{m}(\mathbf{x}, \mathbf{k}, t)$ of the wave-energy density in the wavevector space:

$$
w_{m}(\mathbf{x}, \mathbf{k}, t)=\int_{\Omega_{k}} F_{m}(\mathbf{x}, \mathbf{k}, t) d^{3} k,
$$

where $w_{m}$ is the energy density of a wave mode $m$ observed at a position $\mathbf{x}$ and at a time $t$, and contained in a region $\Omega_{k}$ of wave vectors $k$. Estimation of the experimental signals using a known shape of the WDF constitutes the WDF direct problem. The resolution of this problem is given by Storey and Lefeuvre [1979, 1980], who first solved the problem for waves propagating in a cold plasma without considering the Doppler effect. They have also proposed the use of an explicit frequency dependence of the WDF ((1) contains this dependence in an implicit form, via the plasma dispersion relation which gives the connection between the wave vector and the wave frequency).

Using these results, the inverse problem of the WDF (i.e., the estimation of an approximate shape of the 
WDF based on the knowledge of experimental signals) has been resolved [Lefeuvre, 1977; Lefeuvre and Delannoy, 1979; Delannoy and Lefeuvre, 1986] and applied to the experimental data [e.g., Parrot and Lefeuvre, 1986, Lefeuvre et al., 1992]. More recently, Oscarsson and Rönnmark $[1989,1990]$ and Oscarsson $[1989,1994]$ studied the WDF problem in a hot plasma with the Doppler effect, but using the implicit frequency dependence of the WDF.

The aim of this paper is to develop a method for solving the WDF direct problem in a hot plasma with the Doppler effect, using an approach similar to Storey and Lefeuvre $[1979,1980]$. Section 2 will present the direct problem and the two different approaches, section 3 contains a brief description of our method, whereas applications to real events are given in section 4 . Conclusions are summarized in section 5. (The SI system of units is used throughout the paper.)

\section{The Direct Problem of the WDF}

\subsection{The Different Forms of the WDF}

The original form of the WDF in (1) depends on the wave vector $\mathbf{k}$, which is not easily obtained from experimental measurements. Therefore Storey and Lefeuvre [1979] proposed to replace the wave vector $\mathbf{k}$ by two quantities: the wave-normal direction $\hat{\mathbf{k}}=\mathbf{k} / k(k$ is the modulus of the wave vector), and the observed frequency $\omega_{O}$. The wave-normal direction $\hat{\mathbf{k}}$ is determined by two angles $\theta$ and $\phi$ (Figure 1). For a given wave vector $\mathbf{k}$ the corresponding frequency $\omega_{O}$ is obtained by taking into account the Doppler effect and the response of the medium to wave propagation.

The Doppler effect $\omega_{O}=\left|\omega-\mathbf{k} \cdot \mathbf{v}_{O}\right|,(||$ means the absolute value, $\omega$ is the wave frequency in the medium, and $\mathbf{v}_{O}$ is the velocity of the receiver in the medium) was neglected in the work of Storey and Lefeuvre [1979] where $\omega_{O}=\omega$. This approximation is well justified if the influence of the Doppler effect is negligible in comparison with the experimental frequency resolution. In the following, the velocity $\mathbf{v}_{O}$ is supposed to be much smaller than the velocity of light and nonrelativistic transformations between the receptor and medium frames will be used.

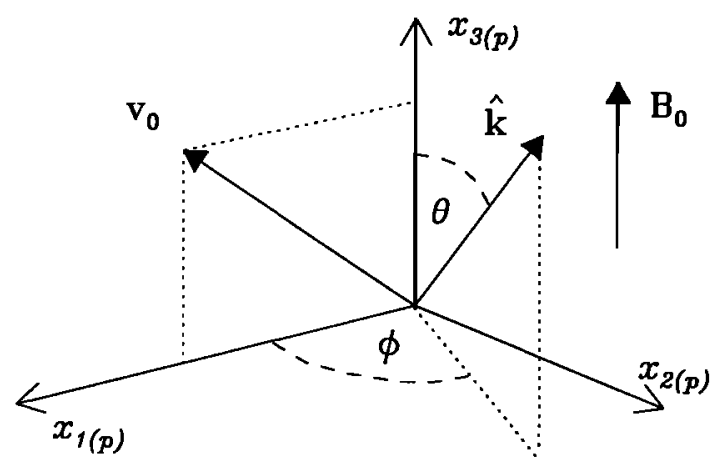

Figure 1. Physical coordinate system $p$ linked with $\mathbf{B}_{0}$ and $\mathbf{v}_{O}$, the latter being the relative velocity between the medium and the satellite.
The response of the medium to the wave propagation is described by the dispersion relation $D(\omega, \mathbf{k})=0$. Using this last substitution, Storey and Lefeuvre [1979], supposing a given position $\mathbf{x}$ and a given time $t$, wrote (1) in a form equivalent to

$$
w_{m}\left(\Omega_{\hat{k}}, \omega_{1}, \omega_{2}\right)=\int_{\omega_{1}}^{\omega_{2}} \int_{\Omega_{\hat{k}}} G_{m}\left(\omega_{O}, \hat{\mathbf{k}}\right) d^{2} \hat{k} d \omega_{O},
$$

where $d^{2} \hat{k}$ represents a two-dimensional integration (over a solid angle) for the wave-normal directions contained in the region $\Omega_{\hat{k}}$. In (1), $\Omega_{k}$ is now defined by the shape of $\Omega_{\hat{k}}$ and the frequency interval $\left(\omega_{1}, \omega_{2}\right)$.

For a given shape of the WDF, i.e., in the case of a pure direct problem, the $G$ form (see (2)) and the $F$ form (see (1)) are equivalent. The WDF can be transformed from one form to the other through the equation

$$
F_{m}(\mathbf{k})=\frac{\left(\mathbf{v}_{g m}-\mathbf{v}_{O}\right) \cdot \hat{\mathbf{k}}}{k^{2}} G_{m}\left(\omega_{O}, \hat{\mathbf{k}}\right)
$$

where $\mathbf{v}_{g m}$ is the group velocity of wave mode $m$. The physical dimension of $F_{m}$ is similar to the energy unit, whereas for $G_{m}$ it is the spectral density of the energy density.

However, the solution of the WDF direct problem is often used as a part of a WDF reconstruction method. The WDF is here defined by experimental data, and the direct problem must be defined more precisely; the task is to calculate an estimation of the data which can be compared with the data really measured. In this case, the two WDF representations are not equivalent. The main advantage of the $G$ form representation (see (2)) is that the $k$ dependence is replaced by the $\omega_{O}$ dependence. The wave data are usually obtained as a function of time, and their $\omega_{O}$ dependence is obtained by spectral analysis. With the $G$ form, a wave phenomenon found around a certain frequency $\omega_{O}$ may be directly compared with a theoretical estimate using the corresponding WDF. Oppositely, the $F$ form requires always a complex $2-D$ surface in the $\mathbf{k}$ space to be found, according to the given $\omega_{O}$. In this case, the only significant WDF values are those obtained on this surface. The same problem arises with the $F$ form in the case of instruments providing spectral information for a predefined set of frequencies [e.g., Cornilleau-Wehrlin et al., 1993].

The $G$ form representation can be therefore assigned to the experimental data in a more straightforward way. As will be shown, a multiplicity of wave modes, often observed in a hot plasma, can be also introduced. The $G$ form has been used by Storey and Lefeuvre [1979], Lefeuvre and Delannoy [1979], and Delannoy and Lefeuvre [1986] to study the reconstruction of the WDF of electromagnetic waves in a cold plasma, without considering the Doppler effect. Storey and Lefeuvre [1979] demonstrated that different wave modes are better separated with this representation.

The $\boldsymbol{F}$ form of the WDF (see (1)) was used by Oscarsson and Rönnmark [1989, 1990] and Oscarsson [1994] to reconstruct the WDF in a hot plasma with the 
WHAMP (Waves in Homogenous, Anisotropic, Multicomponent Plasmas) program [Rönnmark, 1982]. With this last representation, the Doppler effect can be simply introduced.

As discussed by Storey and Lefeuvre [1979], the resolution in frequency $\omega_{O}$ depends always on the temporal resolution of the $G$ form of the WDF. An analogous relation between the wave vector and spatial resolutions must be taken into account for the $F$ form. Rönnmark and Larsson [1988] directly included this relation for their definition of the WDF.

We will use the $G$ form defined in (2), because the final purpose is to reconstruct the WDF from frequencydependent experimental data. In this definition, frequency and time are supposed to be independent variables. The respective resolutions of these variables will be considered as independent experimental errors caused by the spectral analysis of the data.

\subsection{The Relationship Between the WDF and Measurable Wave Parameters}

In the case of electromagnetic waves in plasma, a set of experimental signals $e_{i}$ can be defined as

$$
\mathbf{e}=\left[E_{1}, \ldots, E_{N e}, c B_{1}, \ldots, c B_{N b}, n_{e} \frac{c q_{e}}{\varepsilon_{0} \omega_{O}}\right]
$$

where $E_{1} \ldots E_{N e}$ are the signals obtained by $N_{e}$ electric antennae, $B_{1} \ldots B_{N b}$ are obtained by $N_{b}$ magnetic antennae, $n_{e}$ is the electronic density, $c$ is the velocity of light, $q_{e}$ is the electron charge, and $\varepsilon_{0}$ is the vacuum permittivity. The scale factors for $B_{i}$ and $n_{e}$ are used in order to obtain the same physical unit for all $e_{i}$ components.

For a given frequency $\omega_{O}$ the corresponding spectral matrix is $S_{i j}\left(\omega_{O}\right)=\left\langle\epsilon_{i}\left(\omega_{O}\right) \epsilon_{j}^{*}\left(\omega_{O}\right)\right\rangle$, where $\langle x\rangle$ is the ensemble average of a random variable $x$ and asterisk means the complex conjugate. The $\epsilon_{i}$ are the complex spectrum amplitudes of the experimental signals $e_{i}$. In practice, the spectral analysis roughly corresponds to the filtering of the signal by a passband filter with a central frequency $\omega_{O}$ and a nonzero frequency bandwidth $\Delta \omega$. As discussed by Lefeuvre [1977] and Storey and Lefeuvre [1979], who suppose a continuous distribution of elementary plane waves with no mutual coherence and a narrow bandwidth $\Delta \omega$, the relationship between the spectral matrix and the $G$ form of the WDF is given by

$$
\begin{aligned}
& S_{i j}\left(\omega_{O}\right) \\
& \quad=\sum_{m} \oint a_{m i j}\left(\omega_{O}, \theta, \phi\right) G_{m}\left(\omega_{O}, \theta, \phi\right) d^{2} \hat{k},
\end{aligned}
$$

where $m$ represents the different wave modes. The path integration is carried over the full solid angle of wavenormal directions, and for a given mode $m$ the integration kernels $a_{m i j}$ are calculated from

$$
a_{m i j}\left(\omega_{O}, \theta, \phi\right)=\Delta \omega \frac{\dot{e}_{m i}\left(\omega_{O}, \theta, \phi\right) \dot{e}_{m j}^{*}\left(\omega_{O}, \theta, \phi\right)}{\dot{u}_{m}\left(\omega_{O}, \theta, \phi\right)} .
$$

The quantities $\dot{e}_{i}$ and $\dot{u}$ correspond to an elementary plane wave with a normal direction defined by the angles $\theta$ and $\phi ; \omega_{O}$ represents the Doppler-shifted frequency of the elementary wave; $\dot{u}$ is its energy density; $\dot{e}_{2}$ is the complex amplitude of the $i$ th elementary signal. This amplitude would be equal to the amplitude of the $i$ th experimental signal in the ideal case, where only an elementary plane wave was measured without experimental noise. The complex amplitude $\dot{e}_{i}$ can be calculated by considering the physical properties of the medium, using solutions of the dispersion relation as well as characteristics of the experiment.

\section{Description of the Method}

The resolution of the direct problem of the WDF in a hot plasma is based on (5). The principal task is the calculation of the integration kernels $a_{m i j}$ with (6). In a hot plasma and/or introducing the Doppler effect, a numerical procedure must be employed. This requires a discretization of the integration in (5), and a set of porcupines of distinct wave-normal directions must be introduced. The integration of the WDF direct problem $(5)$ is then transformed into a discrete form for a given frequency $\omega_{O}$,

$$
\begin{aligned}
& S_{i j}\left(\omega_{O}\right) \\
& \quad=\sum_{m} \sum_{\ell=1}^{N_{m}} a_{m i j}\left(\theta_{m \ell}, \phi_{m \ell}\right) G_{m}\left(\theta_{m \ell}, \phi_{m \ell}\right) \sigma_{m \ell}
\end{aligned}
$$

where $\ell$ is the index specifying one of the $N_{m}$ predefined directions for a given porcupine $m$, and $\sigma_{m \ell}$ is the elementary space angle. The quality of reproduction of (5) by the discrete approximation (7) is strongly influenced by the choice of a distribution of directions in a porcupine. We use an igloo distribution (for illustration, see Figure 2) which provides the best coverage of a region of directions (a space angle).

Equation (7) allows a straightforward introduction of different distinct wave modes appearing in a hot plasma

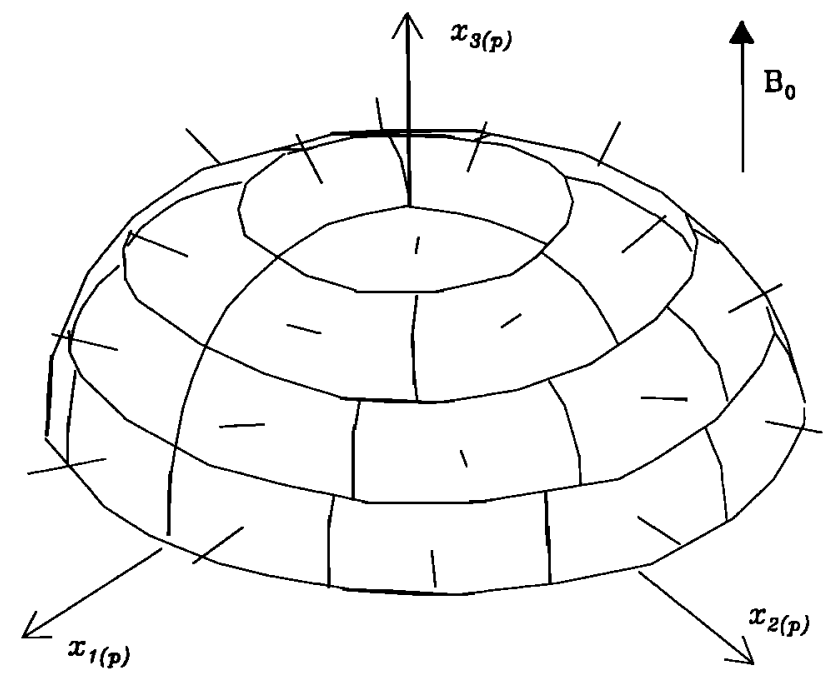

Figure 2. Igloo integration. 
at a given frequency. The index $m$ defines a separate porcupine of directions for each wave mode. The procedure of computing the integration kernels must be therefore able to get all the values of $a_{m i j}\left(\theta_{m \ell}, \phi_{m \ell}\right)$ in the corresponding mode within a porcupine $m$.

To calculate these integration kernels, the dispersion relation of the hot anisotropic medium must be first resolved. Using the $G$ form of the WDF, the Doppler effect will be introduced at this moment, because the frequency $\omega_{O}$ is considered as an independent variable. This requires a simultaneous resolution of the dispersion relation and the equation of the Doppler effect.

\subsection{The Doppler-Shifted Dispersion Relation}

The wave modes are defined by distinct roots of the dispersion relation, which is defined by

$$
D(\omega, \mathbf{k})=\operatorname{det} \mathrm{D}(\omega, k, \theta, \phi)=0,
$$

where the dispersion tensor $D$ is calculated using the dielectric tensor, $E=I+\sum_{l} E_{l} ; l$ is the unit tensor, and $E_{l}$ represents the contribution of a plasma component. The plasma component is defined by the type of particles (electrons or different ion species) or by the shape of their distribution function. The following three different approximations of $E_{l}$ have been included in our method: cold plasma, algebraic expressions for a hot and weakly relativistic plasma [Sazhin, 1987, 1988], and a numeric procedure for a hot nonrelativistic plasma [Rönnmark, 1983]. Following Sazhın [1987], the nonrelativistic approximation does not allow the analysis of plasma waves near the cutoff frequencies, where the value of the refractive index is small.

The equation of the Doppler effect $\Re \omega=\omega_{O}+\mathbf{k} \cdot \mathbf{v}_{O}$ must be used with (8) to find $k$ and $\Im \omega$ for a given value of $\omega_{O}$ and a given wave-normal direction. The nonlinear system of equations can have several solutions corresponding to different wave modes.

Several cases must be considered to obtain the solution of the dispersion relation, depending on the stability of the wave mode. If the wave is stable, $\Im \omega$ is null and $E$ is a Hermitian tensor [Stix, 1962]. Therefore D also is a Hermitian tensor and its determinant is real. The solution is given by the real roots $k$ as soon as the wave-normal direction and the frequency are fixed. If the wave modes are quasi-stable; that is, $\Im \omega$ is low, approximations can be used (for example, [Sazhin, 1993]). For a more general solution, a combination of $k$ and $\Im \omega$ that makes the complex value $\operatorname{det} \mathrm{D}(\omega, k, \theta, \phi)$ to vanish for given $\theta, \phi$, and $\omega_{O}$ must be found. As the resolution of the dispersion relation is a numerical problem, it is necessary to make a preliminary estimate of the solution for wave mode $m$ in an initial wave-normal direction.

Generally, the dispersion relation is symmetrical in the space of wave-normal directions and the solution can only be sought in a part of this space. However, the symmetry is disturbed by the Doppler effect, and several cases must be envisaged, depending on the value of the $\mathbf{v}_{O}$ components.

\subsection{The Integration Kernels}

Using the solution of the dispersion relation, the integration kernels are calculated. In (6) the ratio for a given mode $m$ can be written as (supposing given $\omega_{O}$, $\theta$, and $\phi)$

$$
\frac{\dot{e}_{i} \dot{e}_{j}^{*}}{\dot{u}}=\dot{e}_{i}^{n} \dot{e}_{j}^{n *},
$$

where

$$
\dot{e}_{i}^{n}=\frac{\dot{e}_{i}}{\sqrt{\dot{u}}} .
$$

In the definition (9b) of the normalized vector $\dot{\mathbf{e}}^{n}$, the vector é of analytic signals must correspond to the vector of experimental signals given by (4). To obtain $\dot{e}^{n}$, the parameters of the elementary plane wave (electric and magnetic fields, electronic density variation) will be found first. In the second step the influence of experimental devices (electric or magnetic antennae) will be considered.

For a given wave mode $m$ the solution of the dispersion relation can be used to calculate the dispersion tensor $D_{m}$. The polarization of the electric field $\mathbf{E}$ of the elementary plane wave, which is necessary to calculate the kernels $a_{m i j}$, can be found from $D_{m} \cdot \dot{\mathbf{E}}=0$. With the polarization and the following expression of the energy density of the analytic plane wave which can be derived from Stix [1962],

$$
\dot{u}=\frac{1}{4} \omega \varepsilon_{0} \dot{\mathbf{E}}^{*} \cdot \frac{\partial \mathrm{D}_{m}}{\partial \omega} \cdot \dot{\mathbf{E}},
$$

a direct procedure to calculate the normalized electric field $\dot{\mathbf{E}}^{n}=\dot{\mathbf{E}} / \sqrt{\dot{u}}$ has been found. The normalized wave magnetic field is then given by

$$
\dot{\mathbf{B}}^{n}=c \dot{\mathbf{B}} / \sqrt{\dot{u}}=\frac{c}{\Re \omega} \mathbf{k} \times \dot{\mathbf{E}}^{n}
$$

and the normalized electronic density perturbation $\dot{n}_{e}^{n}$ is linked to the normalized electric field $\dot{\mathbf{E}}^{n}$ through Coulomb's law:

$$
\dot{n}_{e}^{n}=\frac{c}{\omega_{O}} \mathbf{k} \cdot \dot{\mathbf{E}}^{n}
$$

The normalized signals $\mathrm{e}^{n}$ can now be calculated, using the results of the last paragraphs. As for the signals of the electric and magnetic components, we must calculate the projection of the field vector in the direction of the corresponding antenna. The electric signals may need a supplementary correction to account for the finite length of the antenna and the coupling impedance to the plasma. As the next step, all signals are multiplied by the complex transfer function of the corresponding electronic chain. Finally, with the signals $\mathbf{e}^{n}$ and $(9 \mathrm{a})$, we can calculate the kernels $a_{m i j}$.

\subsection{The Group Velocity}

When the dispersion relation is known for a given wave mode $m$, the group velocity of an elementary plane wave can be calculated as 


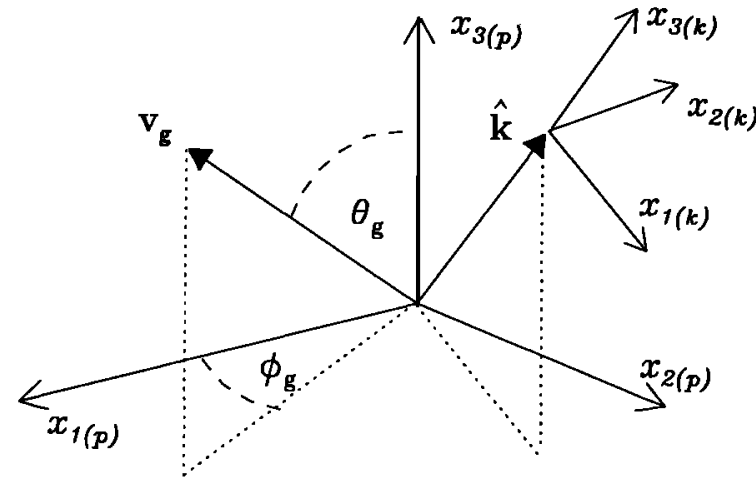

Figure 3. Coordinate system $k$ linked to the wave vector.

$$
v_{g m}=-\Re\left(\frac{\partial_{k} \operatorname{det} \mathrm{D}_{m}}{\partial_{\omega} \operatorname{det} \mathrm{D}_{m}}\right) .
$$

The calculation of this velocity is necessary for the transformation from one representation of the WDF to the other with (3). It could also be useful for interpreting the results of the WDF analysis.

Using the derivatives of $\mathrm{D}_{m}$, we have

$$
\begin{aligned}
& v_{g 1(k)}=-\Re\left(\frac{\partial_{\theta} \operatorname{det} \mathrm{D}_{m}}{k \partial_{\omega} \operatorname{det} \mathrm{D}_{m}}\right), \\
& v_{g 2(k)}=v_{O 1(p)} \sin \phi, \\
& v_{g 3(k)}=-\Re\left(\frac{\partial_{k} \operatorname{det} \mathrm{D}_{m}}{\partial_{\omega} \operatorname{det} \mathrm{D}_{m}}\right),
\end{aligned}
$$

where the derivative $\partial_{\phi}$ det $D_{m}$ is found from the fact that $\operatorname{det} D_{m}$ depends only on the angle $\phi$ through the Doppler effect. The quantity $v_{O 1(p)}$ is the component of the velocity $\mathbf{v}_{O}$ in the direction of the axis $x_{1(p)}$ (see Figure 1).

The components of the group velocity are calculated in the coordinate system linked to the wave vector ( $\mathrm{Fi}$ gure 3 ). To express the group velocity in the physical coordinate system of Figure 1, a transformation has to be performed.

\section{Examples of Solutions}

\subsection{Whistler Mode Waves in a Plasma Near the Geostationary Orbit}

The first tests of this method used typical plasma parameters near the equator at $L=6.6$. Two main reasons lead to this choice. (1) In this region a twopeaked WDF of whistler-mode waves (i.e., violations of the assumption of a single plane wave) has been observed on board Geos 1 [Parrot and Lefeuvre, 1986]. (2) The work of Horne and Sazhin [1990] and Sazhin and Horne [1990] contains a numerical solution of the dispersion relation for the whistler-mode waves at fixed frequencies. Their plasma model corresponds to Geos 1 observations in this region. To compare their results with the output of our method, the same plasma and wave parameters have been chosen.

The plasma model is composed of the following three Maxwellian components: (1) cold electrons with a number density of $10^{6} \mathrm{~m}^{-3}$ and temperature $T=1 \mathrm{eV}$; (2) hot electrons with a density of $1.81 \times 10^{5} \mathrm{~m}^{-3}$ and $T=$ $200 \mathrm{eV}$; and (3) ions as a neutralizing background. The electron gyrofrequency is $\Omega_{e} / 2 \pi=3000 \mathrm{~Hz}$.

Three whistler-wave frequencies have been examined as follows: $\omega_{O} / 2 \pi=1200,1800$, and $2400 \mathrm{~Hz}$. No Doppler effect has been introduced $\left(\omega_{O}=\Re \omega\right)$. Figure 4 contains an example of results in the case of $\omega_{O} / 2 \pi=$ $1800 \mathrm{~Hz}$. The dependencies of obtained parameters on the polar angle $\theta$ are presented. Without the Doppler effect the dependencies on the azimuthal angle $\phi$ are either constant functions or can be determined as simple algebraic expressions. The value $\phi=0^{\circ}$ has been therefore used.

Figure 4 (left) contains (from the top) the waverefractive index, $N=k c / \Re \omega$ ( $c$ is the velocity of light); the ratio $\Im \omega / \Re \omega$ expressing wave damping; the groupvelocity modulus normalized to the velocity of light $v_{g} / c$; and the group-velocity direction represented by the deviation of the group-velocity vector from the vector of the ambient magnetic field. The solid line corresponds to the solutions obtained when using the numeric procedure for the hot-plasma dielectric tensor [Rönnmark, 1983], the dotted line corresponds to algebraic expressions of this tensor [Sazhin, 1987, 1988], and the dashed line represents the cold-plasma approximation of the dielectric tensor [Stix, 1962]. We have calculated the cold-plasma solution taking into account the total plasma density obtained as the sum of the densities of both cold and hot electrons. For $\theta$ well below the resonance angle $\theta_{R}$, the three procedures give similar results (in the presented case $\theta_{R}=51.8^{\circ}$ ), the observed differences between the hot and cold plasma results being less than $2 \%$. However, if a simplified cold plasma solution is used, the bias of the obtained refractive index can reach about $10 \%$ [Sazhin and Horne, 1990]. In the cold-plasma approximation the waves do not propagate for $\theta$ greater than $\theta_{R}$.

Considering the results of the hot-plasma procedure, we observe a complex behavior of the wave propagation near the resonance angle and a heavy damping for large $\theta$ values, $\theta>57^{\circ}$. The plots of the hot-plasma refractive index obtained by a numerical procedure of Horne and Sazhin [1990] and Sazhin and Horne [1990] have been well reproduced by our procedure when using the numerical approximation of the dielectric tensor. The same holds true for the other two frequencies, 1200 and $2400 \mathrm{~Hz}$ (not shown). Additionally, new branches of the dispersion curves have been found near the resonance angle. All presented curves are composed of four distinct parts and were created by independent runs of the procedure for solving the dispersion relation. For each run the initial refractive index $N$ was found analyzing all solutions of the dispersion relation for $N<1000$. For $\theta>\theta_{R}$ the differences between the results of numeri- 

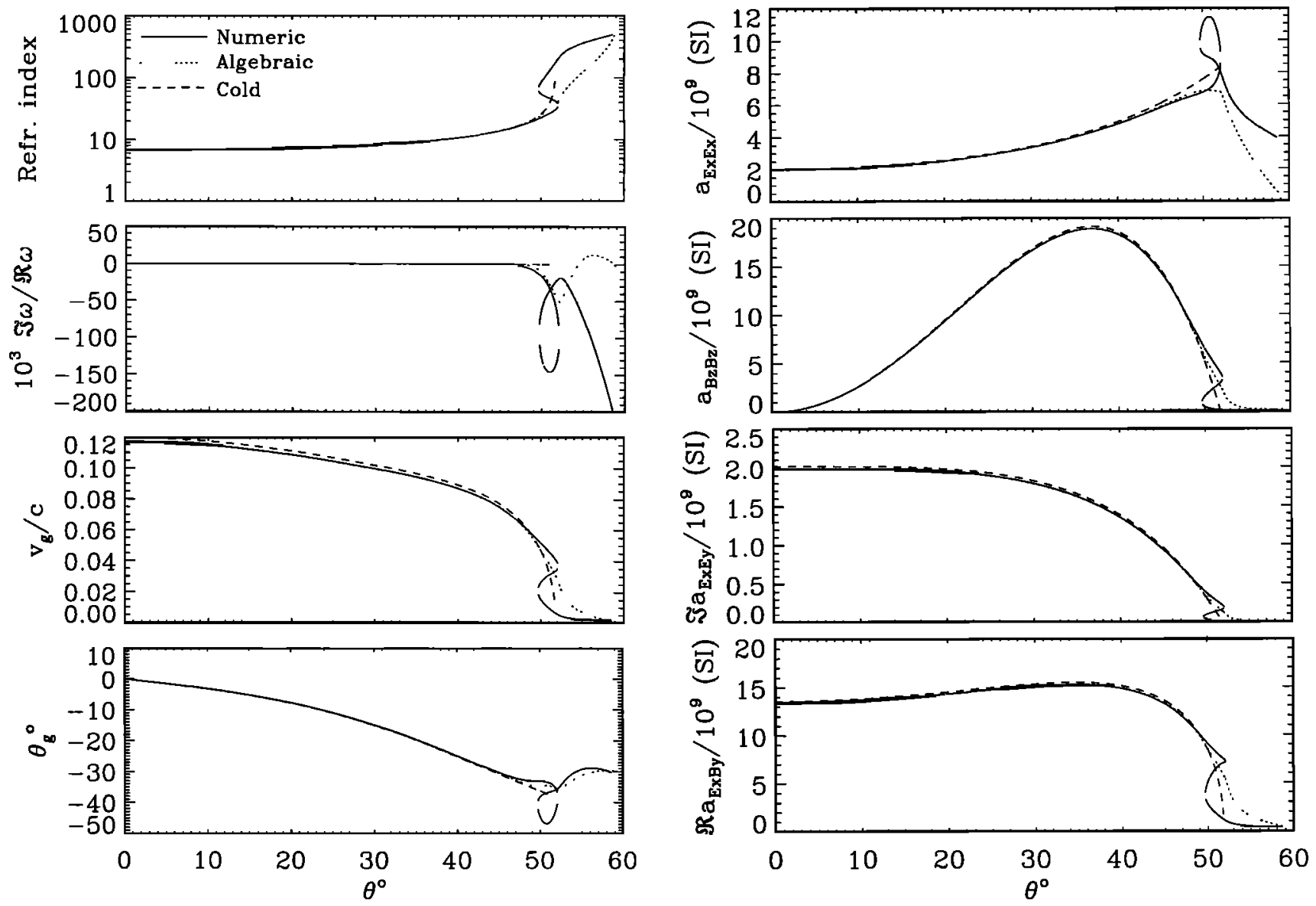

Figure 4. Solution to the wave distribution function (WDF) direct problem for equatorial hiss outside the plasmasphere. The frequency is $1800 \mathrm{~Hz}$ and corresponds to $0.6 \Omega_{e} / 2 \pi$. A plasma model containing $18.1 \%$ of Maxwellian electrons with a temperature of $200 \mathrm{eV}$ is assumed. The results obtained with three different procedures for the plasma dielectric tensor are compared. (left) Dependence of the solution of the dispersion relation on $\theta$, refractive index, wave damping, and the group velocity vector of an elementary whistler wave. (right) Dependence of some integration kernels $a_{i j}$ calculated for the same elementary wave on $\theta$.

cal and algebraic procedures are presented in Figure 4. The wave growth obtained by the algebraic procedure is unrealistic, because the validity of the algebraic approximation fails for $\theta>52.8^{\circ}$, according to the conditions stated by Sazhin [1987, 1988].

As has been shown, the calculation of the spectra estimates for a given WDF is straightforward once the values of the integration kernels are known (see (7)). For example, a WDF shape modeled by several plane waves can be introduced by the summation of only the terms corresponding to the respective wave-normal directions. If the WDF shape is given as an analytic function, the spectra estimation represents a simple summation of a large number of known terms. The knowledge of integration kernels is therefore crucial to solve the WDF direct problem. In Figure 4 (right), examples of the $\theta$ dependencies of some integration kernels $a_{i j}$ are presented. Three magnetic and three electric antennae were used, with their directions along the axes of the coordinate system of Figure 1. The electric field $\left(E_{x}, E_{y}\right.$, and $\left.E_{z}\right)$ and magnetic field components, rescaled by the velocity of light $\left(c B_{x}, c B_{y}\right.$, and $\left.c B_{z}\right)$, have been used in place of the experimental signals $\dot{e}_{i}$ in (6). The SI units have been used to calculate absolute values of the kernels; that is, their physical dimension is $\left[\mathrm{V}^{2} \mathrm{~m} \mathrm{~J}^{-1} \mathrm{~Hz}\right]$. Four representative examples of the kernels are drawn. The dependencies calculated with the cold-plasma approximation of the dielectric tensor are equal to the results of the algebraic expressions derived by Storey and Lefeuvre [1980]. They are also approximately equal to the hot-plasma solutions. For $\theta$ values well below the resonance angle, the difference is less than $2 \%$. Comparing the results of the two hot-plasma procedures (numeric and algebraic), an excellent conformity is obtained, except for $\theta$ values above $50^{\circ}$, where a complex behavior of the numerical hot-plasma dependencies is observed. The simpler algebraic approximation cannot be used so near the resonance cone. However, this restriction is not very important, because it applies only in the case of heavily damped waves.

The results indicate that the hot-plasma approach may be useful in the study of equatorial hiss outside the 
plasmasphere. Hot electrons convected from the plasma sheet can influence the relative amplitudes and phases of observed waves over a range of several degrees near the resonance cone. Therefore the shape of the integration kernels $a_{i j}$ is biased against the cold-plasma case and the reconstruction of the WDF should take this effect into account, especially if the waves are found near the resonance angle.

The existence of several whistler-mode branches in a narrow $\theta$ interval near the resonance angle may be a problem arising from the hot-plasma approach; for a given wave-normal direction there are several values of the integration kernels and the WDF reconstruction becomes more complex. In the particular case of Figure 4 the rejection of the heavily damped branches is a natural way to solve the problem. The characteristic length of wave damping of these branches can be estimated by using the presented results; the satellite must be at a distance of less than several tens of kilometers from the region of generation to observe the damped whistler branches. However, if several branches with a comparable damping rate are present (as was observed for $\omega_{O} / 2 \pi=2400 \mathrm{~Hz}$ ), this straightforward solution cannot be applied and a more complex multimodal WDF reconstruction is necessary.

\subsection{Ion-Cyclotron Waves in the Auroral Magnetosphere}

Another example is taken from a different region of the Earth's magnetosphere. On the auroral field lines, wave propagation and stability can be affected by beams of energetic electrons. Alfvèn electromagnetic ion cyclotron (EMIC) waves are often observed simultaneously with electron precipitation [e.g., Temerin and Lysak, 1984]. Oscarsson and Rönnmark [1990] studied an EMIC wave emission observed on board the Viking satellite at an altitude of $4300 \mathrm{~km}$. The study is based on the data of two electric antennae and follows the procedure of Oscarsson and Rönnmark [1989]. The main part of the observed wave energy was concentrated around half of the local proton gyrofrequency. The observed auroral electrons are modeled by a drifting Maxwellian distribution, with a drift energy of $4 \mathrm{keV}$ and the relative density $0.1 \%$ of cold proton-electron plasma.

The published solutions of the WDF direct problem correspond to the wave-vector-dependent representation. A limited range of wave-vector components near the resonance of EMIC waves is taken into account. This solution has been compared with the output of our method. A range of frequencies from $19 \mathrm{~Hz}$ to the proton gyrofrequency $\Omega_{\mathrm{H}^{+}} / 2 \pi=190 \mathrm{~Hz}$ and a range of $\theta$ values from $80^{\circ}$ to $89.9^{\circ}$ have been examined. The principal results in a selected part of this range are shown in Figure 5. The spatial growth $\zeta=\Im \omega / 2 \pi v_{g}$ is presented as a 3-D image in the gray scale. White corresponds to strongly damped waves out of the resonance cone, and the gray-to-black scale represents stable and growing waves. Three isolines provide rough information about values of the refractive index $N$. They are

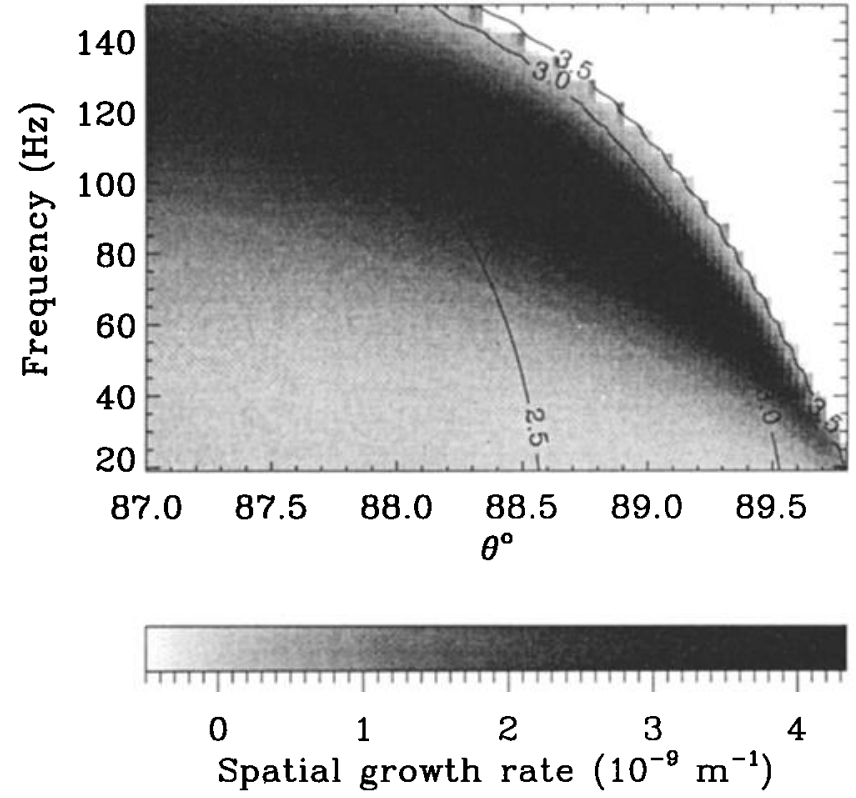

Figure 5. Solution of the hot-plasma dispersion relation in the auroral region for an altitude of $4300 \mathrm{~km}$. The hot-plasma model as in the work of Oscarsson and Rönnmark [1990] has been used. The gray scale corresponds to the spatial-growth coefficient $\Im \omega / 2 \pi v_{g}$, plotted versus wave frequency $\omega_{O}$, and the angle $\theta$ between the wave-normal and the ambient magnetic field. The labeled isolines represent the common logarithm of the refractive index.

labeled by the common logarithm of $N$; that is, they correspond to $N=316,1000$, and 3160 . In the region shown the minimum value is $N=150$ for $\theta=87^{\circ}$ and $\omega_{O} / 2 \pi=20 \mathrm{~Hz}$. A flat maximum of the spatial growth is found at $\theta \approx 88.6^{\circ}$ and at frequency $\omega_{O} / 2 \pi \approx 100 \mathrm{~Hz}$, which agrees with the observed maximum of wave activity. The corresponding refractive index is $N \approx 470$. To compare these results with the original solution of Oscarsson and Rönnmark [1990], a transformation was made to the space of wave vectors. The published results are well reproduced, including the absolute values of the wave-growth coefficient.

Note that the obtained maximum wave growth of 4.3 $\times 10^{-9} \mathrm{~m}^{-1}$ is too small to explain the observations. The amplification of the wave amplitude by a factor of 7 is needed to get the observed signal-to-noise ratio. With a spatial growth rate of $4.3 \times 10^{-9} \mathrm{~m}^{-1}$ the waves must propagate across a distance of $7.2 \times 10^{7} \mathrm{~m}$, which corresponds to an unrealistic distance of about 11 Earth radii. A wrong assumption may have been made about the form of the distribution function of the electron beam in the generation region. Gustafsson et al. [1990] noted that the wave growth may be increased by a higher density and smaller thermal spread of the model beam. The approximative calculation of Temerin and Lysak [1984] provides a higher spatial growth for a smaller drift energy of the beam. As will be confirmed by our numeric results, an increased beam density and decreased thermal and drift energy lead to higher wave 
growths, but the propagation characteristics of EMIC waves become more complex.

Erlandson et al. [1994] recently studied the auroral region at lower altitudes (about $1800 \mathrm{~km}$ ) using the data of the Freja satellite. EMIC waves were observed in the region of electron precipitation at an invariant latitude of $69^{\circ}$. An estimation provides a low plasma electron density of $40 \mathrm{~cm}^{-3}$. Wave emissions were confined to the following two frequency bands: (1) lowfrequency waves below the local oxygen gyrofrequency $\left(\Omega_{\mathrm{O}+} / 2 \pi \approx 25 \mathrm{~Hz}\right)$, with a maximum activity at $8 \mathrm{~Hz}$, and (2) higher-frequency waves, between the local he$\operatorname{lium}\left(\Omega_{\mathrm{He}^{+}} / 2 \pi \approx 100 \mathrm{~Hz}\right)$ and hydrogen gyrofrequencies, peaked at about $130 \mathrm{~Hz}$.

No analysis of wave propagation and stability was made for this event, and we have applied the developed method to this case for the following reasons. (1) Using the solution to the dispersion relation of EMIC waves, we can deduce the main properties of wave propagation and we can attempt to explain the observed spectrum. (2) The possibility and usefulness of the WDF reconstruction for similar events can be verified.

The propagation characteristics of EMIC waves are strongly influenced by the relative abundance of ion species. We thus need this information as input data for the resolution of the WDF direct problem. As direct measurement of the thermal plasma composition
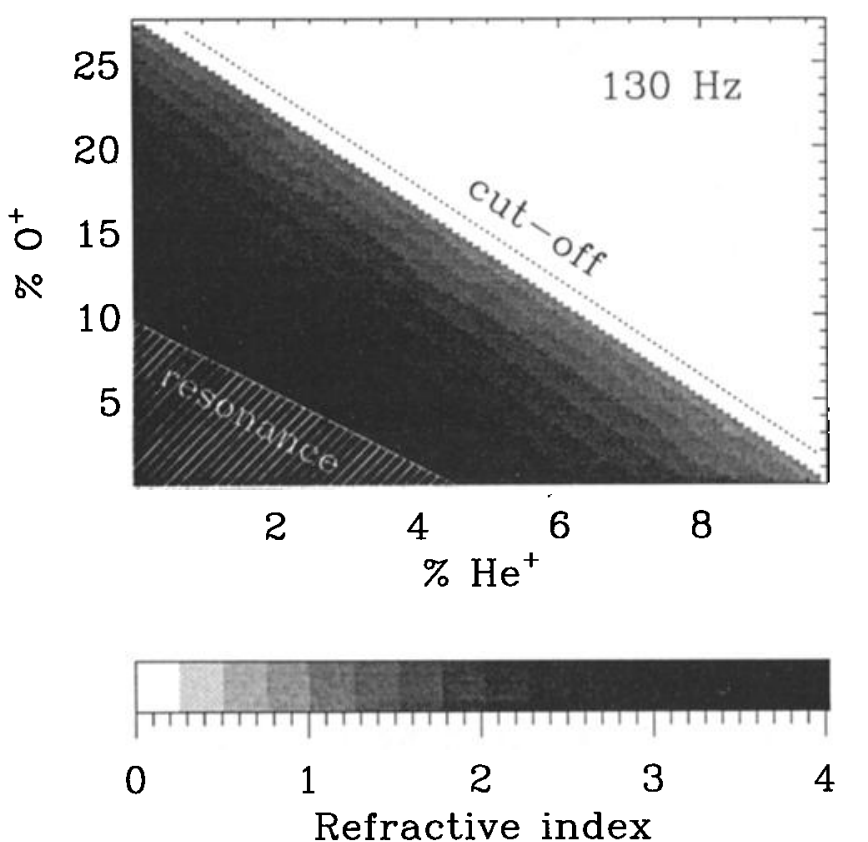

Figure 6. The refractive index of electromagnetic ion cyclotron (EMIC) waves in the higher-frequency band of the event described by Erlandson et al. [1994] versus the relative abundance of helium and oxygen ions. The scale corresponds to $\theta=0^{\circ}$. The following three regions with different qualitative properties of wave propagation are shown (from top right to bottom left): evanescent waves, unducted waves without plasma resonance, and ducted waves with a resonance near $\theta=90^{\circ}$. The plasma was assumed to be cold. has not been published by Erlandson et al. [1994], we will use the wave observations as an estimator of these data. The ion composition mainly affects the higherfrequency band of observed EMIC waves. We will first suppose a plasma composed of cold hydrogen, helium, and oxygen ions and cold electrons. Figure 6 shows a view of the propagation of left-polarized EMIC waves at $130 \mathrm{~Hz}$ for various ion compositions. The scale of gray shades represents the value of the refractive index $N$ for $\theta=0^{\circ}$. In the region above the diagonal the mode is evanescent and the waves do not propagate in a plasma with higher oxygen and helium fractions. The dotted line represents the cutoff $(N=0)$. From the cutoff to the bottom left corner (where $100 \%$ of $\mathrm{H}^{+}$is assumed), the refractive index increases up to $N=4$. The oblique, light-colored boundary line corresponds to the ion composition where the crossover frequency is equal to $130 \mathrm{~Hz}$. On this line, two cold-plasma modes are exchanged. The crossover line bounds a region where the waves have a plasma resonance $(N \rightarrow \infty)$ near $\theta=90^{\circ}$. Outside of this region, there is no resonance of EMIC waves. The waves with resonance have the direction of group velocity nearly always aligned with the ambient magnetic field. The waves without resonance have the group velocity roughly aligned with the phase velocity; that is, the group velocity has the direction of the DC magnetic field only for $\theta \approx 0^{\circ}$.

The stopband in the measured spectra [Erlandson et al., 1994] between the local $\mathrm{He}^{+}$and $\mathrm{O}^{+}$gyrofrequencies is an important feature of the event. A more detailed analysis of the wave propagation shows that the observed spectrum implies a negligible fraction of $\mathrm{He}^{+}$ ions. If only $1 \%$ of helium were supposed, the wave propagation below $\Omega_{\mathrm{He}^{+}}$would be possible in a bandwidth of $\frac{1}{4} \Omega_{\mathrm{He}^{+}}$, and a new stopband above $\Omega_{\mathrm{He}^{+}}$would be induced. Nevertheless, the presence of $\mathrm{O}^{+}$and $\mathrm{H}^{+}$ fractions is required to explain the observed stopband. Its lower boundary is then defined by the local oxygen gyrofrequency. If the higher frequency band contains left-polarized EMIC waves, the boundary at $100 \mathrm{~Hz}$ can be explained in two different ways.

1. The first is the cutoff of EMIC waves. The corresponding ion-composition estimate is $20 \%$ of $\mathrm{O}^{+}$and $80 \%$ of $\mathrm{H}^{+}$. The EMIC waves above $100 \mathrm{~Hz}$ propagate in a mode without plasma resonance. The frequency interval where the theory predicts the impossibility of propagation of EMIC waves corresponds to the observed stopband.

2. The second is the crossover of EMIC waves. This corresponds to a composition with $5 \%$ of $\mathrm{O}^{+}$and $95 \%$ of $\mathrm{H}^{+}$. The EMIC waves above $100 \mathrm{~Hz}$ have a plasma resonance, and their group velocity is directed along the field lines.

Figure 7 presents the solution to the WDF direct problem for a higher band of observed EMIC waves (a representation similar to that in Figure 4 has been used); a frequency of $130 \mathrm{~Hz}\left(\approx \frac{1}{3} \Omega_{\mathrm{H}^{+}}\right)$and a plasma with $95 \%$ of cold $\mathrm{H}^{+}$and $5 \%$ of cold $\mathrm{O}^{+}$have been supposed. The model plasma composition follows case 2 of the analysis presented above. However, the majority 

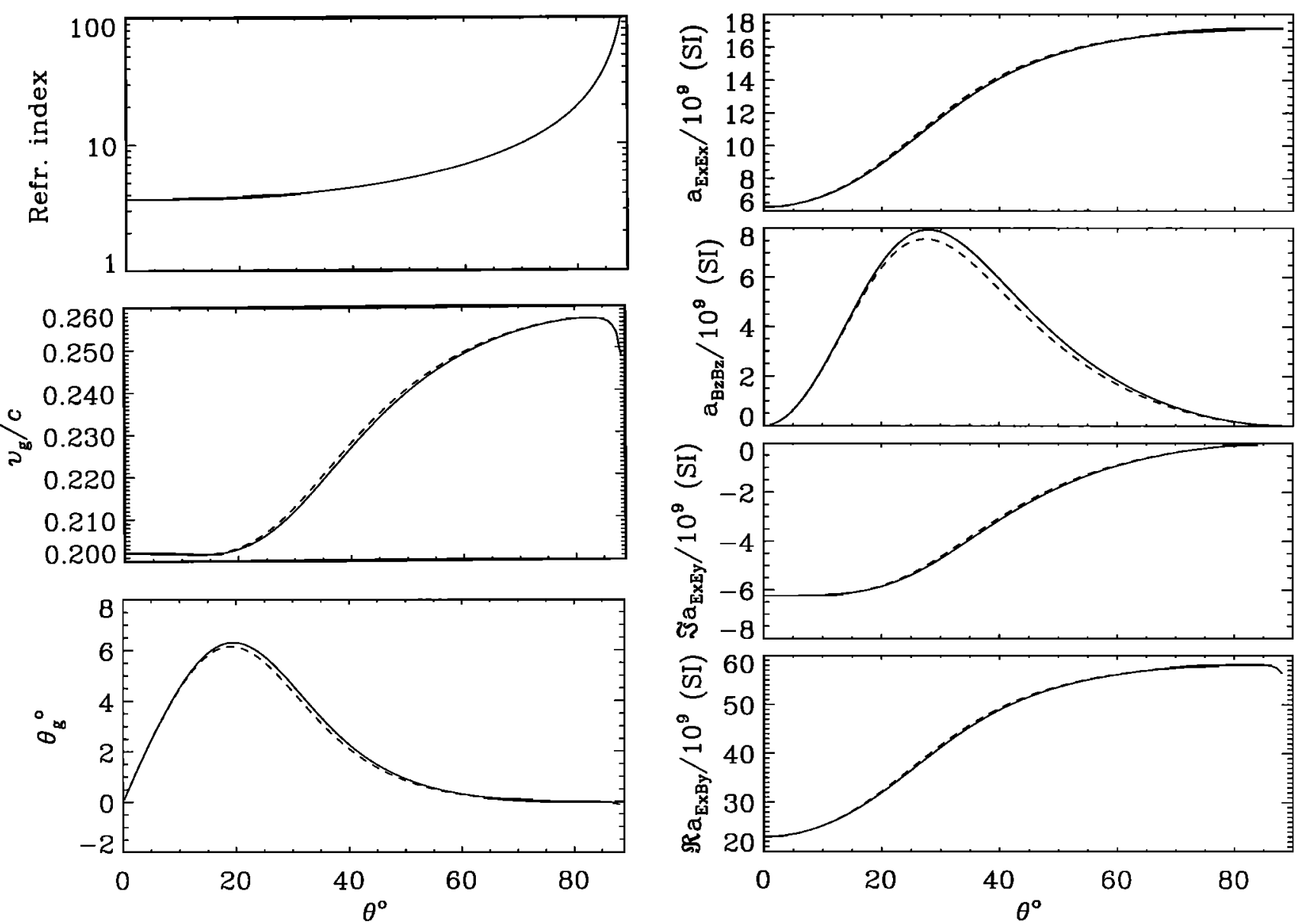

Figure 7. Example of the solution of the WDF direct problem for ducted EMIC waves in the auroral region. The observed frequency of $130 \mathrm{~Hz}\left(\approx \frac{1}{3} \Omega_{\mathrm{H}^{+}} / 2 \pi\right)$ is assumed. The dashed line represents the cold-plasma approximation of the dielectric tensor, and the solid line corresponds to numeric results involving auroral electron beams. (left) Dependence of the solution of the dispersion relation on $\theta$, refractive index, group-velocity modulus normalized to the velocity of light, and deviation of the group velocity from the ambient magnetic field. (right) Several integration kernels $a_{i j}$ in SI units versus $\theta$.

of $\mathrm{H}^{+}$is unusual at low altitudes where the observations have been made [e.g., see Lefeuvre et al., 1992]. In Figure 7 the cold-plasma approximation of the electron distribution (dashed line) is compared with the hotplasma numeric results (solid line) in the whole range of $\theta$ values. The hot-plasma model was chosen in accordance with the electron-energy spectra published by Erlandson et al. [1994]. A low temperature of $1 \mathrm{eV}$ was assumed for the main Maxwellian component of electron distribution. Maxwellian electrons with higher temperature are not apparent in the spectra. Two drifting Maxwellian beams with drift energies of $1 \mathrm{keV}$ and $100 \mathrm{eV}$ have been added. They propagate earthward in the direction parallel to the ambient magnetic field. The energy corresponding to the thermal spread of the beams has been fixed at 0.1 of the respective drift energy. The published spectra are not calibrated to the absolute values of electron flux. The relative number of electrons in the beams must therefore be treated as a free parameter. A relatively high fraction of beam elec- trons has been assumed to emphasize hot-plasma effects on the wave propagation; each beam is taken to contain $1 \%$ of the total electron density. Figure 7 shows a slight difference between the hot- and cold-plasma solutions, especially in the $\theta$ range from $20^{\circ}$ to $60^{\circ}$. The wave dispersion expressed by the refractive index is practically not affected by this difference, whereas the group velocity and integration kernels exhibit a small bias of several percent (up to $8 \%$ in the case of kernel $a_{B z B z}$ ). All differences are due to the electron beams. The replacement of the 1-eV Maxwellian electrons by their cold-plasma approximation has a negligible effect on the obtained results as well as the use of 1-eV Maxwellian ions in place of the cold ones.

A surprising effect is that the electron beams influence wave propagation relatively far from the resonance, where no effects concerning the wave stability occur. More pronounced hot-plasma effects over a wider range of $\theta$ values might be supposed in the case of a substantial hot Maxwellian fraction in the electron distri- 

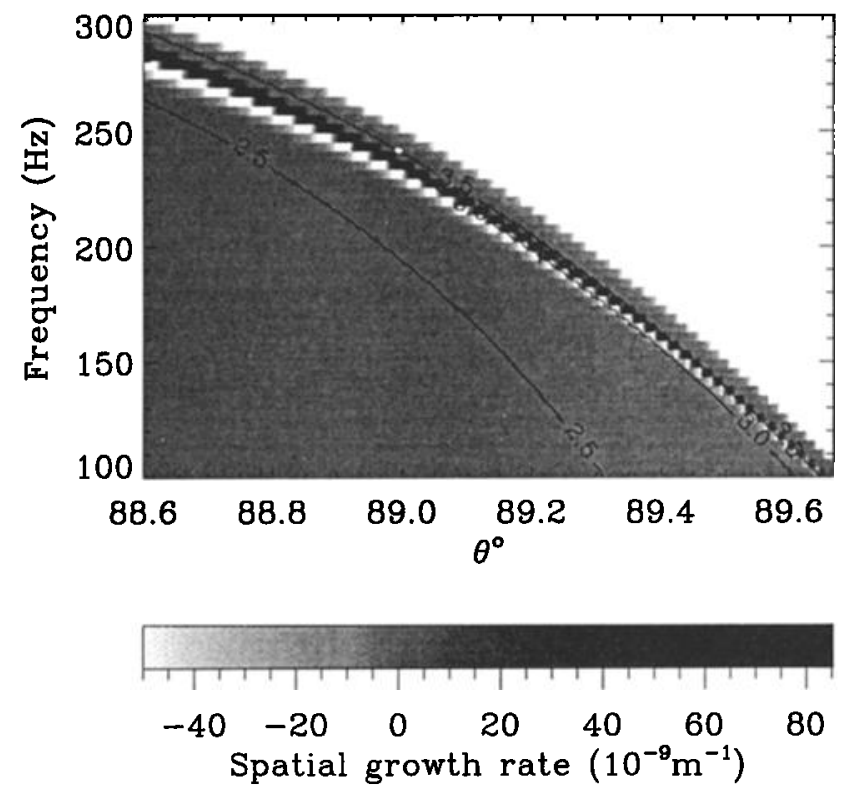

Figure 8. Solution of the hot-plasma dispersion relation of ducted EMIC waves at an altitude of $1700 \mathrm{~km}$. The plasma model consists of $95 \%$ of cold $\mathrm{H}^{+}$ions, $5 \%$ of cold $\mathrm{O}^{+}$ions, $99.9 \%$ of Maxwellian electrons with a temperature of $1 \mathrm{eV}$, and $0.1 \%$ of auroral electrons forming a beam with the drift energy $1 \mathrm{keV}$ and thermal energy $100 \mathrm{eV}$. The spatial growth rate is plotted as a two-dimensional function of the frequency and $\theta$. Three isolines correspond to the wave refractive indices 316,1000 , and 3160 and are labeled by the common logarithms of these values.

bution. Such a distribution has been reported, e.g., by Gustafsson et al. [1990] from orbit 849 of the Viking satellite, where over $10 \%$ of the total electron density is observed as Maxwellian electrons with a temperature of $500 \mathrm{eV}$. Our calculations show that the influence on the obtained curves is negligible, even if $20 \%$ of $500 \mathrm{eV}$ electrons are assumed to be present.

Near the resonance angle, the auroral electrons can exchange their energy with the EMIC waves. The $\theta$ interval where unstable waves can be found is very narrow, below the resolution of Figure 7. The solution for the dispersion relation has been used to analyze wave stability near the resonance. Figure 8 shows the solution to the complex dispersion relation as in Figure 5 . The spatial growth rate is presented in the frequency$\theta$ plane by shades of gray. Approximate information about the values of the wave refractive index is given by isolines. The plasma model consists of cold ions ( $95 \%$ $\mathrm{H}^{+}$and $5 \% \mathrm{O}^{+}$), Maxwellian electrons with a temperature of $1 \mathrm{eV}$, and a single electron beam with the drift energy $1 \mathrm{keV}$. The energy of the thermal spread has been assumed to be 0.1 of drift energy, and beam electrons are supposed to constitute $0.1 \%$ of the total electron density. A narrow interval of $\theta$ values of about $1^{\circ}$ near the resonance is examined. The range of frequencies covers the observed spectral peak from $\frac{1}{4} \Omega_{\mathrm{H}}+$ to $\frac{3}{4} \Omega_{\mathrm{H}^{+}}$. A fine step-like structure is an artifact due to a finite number of examined frequencies. A light region in the top right part of Figure 8 corresponds to heavily damped waves outside the resonance cone. At a fixed frequency and with decreasing $\theta$ we first see a short, flat interval of relatively stable waves, then a sharp positive peak of growing waves, and suddenly, a deep negative peak of wave damping. For low $\theta$ values the waves are stable (represented by the gray area at the bottom left of Figure 8) and the wave parameters are not far from the cold-plasma approximation as shown in Figure 7. For an increasing frequency the maximum spatial growth rate increases from about $3 \times 10^{-8} \mathrm{~m}^{-1}$ to over $8 \times 10^{-8} \mathrm{~m}^{-1}$, contrary to the trend observed in the measured spectrum, where the maximum intensity falls in lower frequencies. The absolute values of wave growth are higher than in the case of the EMIC waves in Figure 5, but they are still insufficient to explain the observed signal-to-noise ratio.

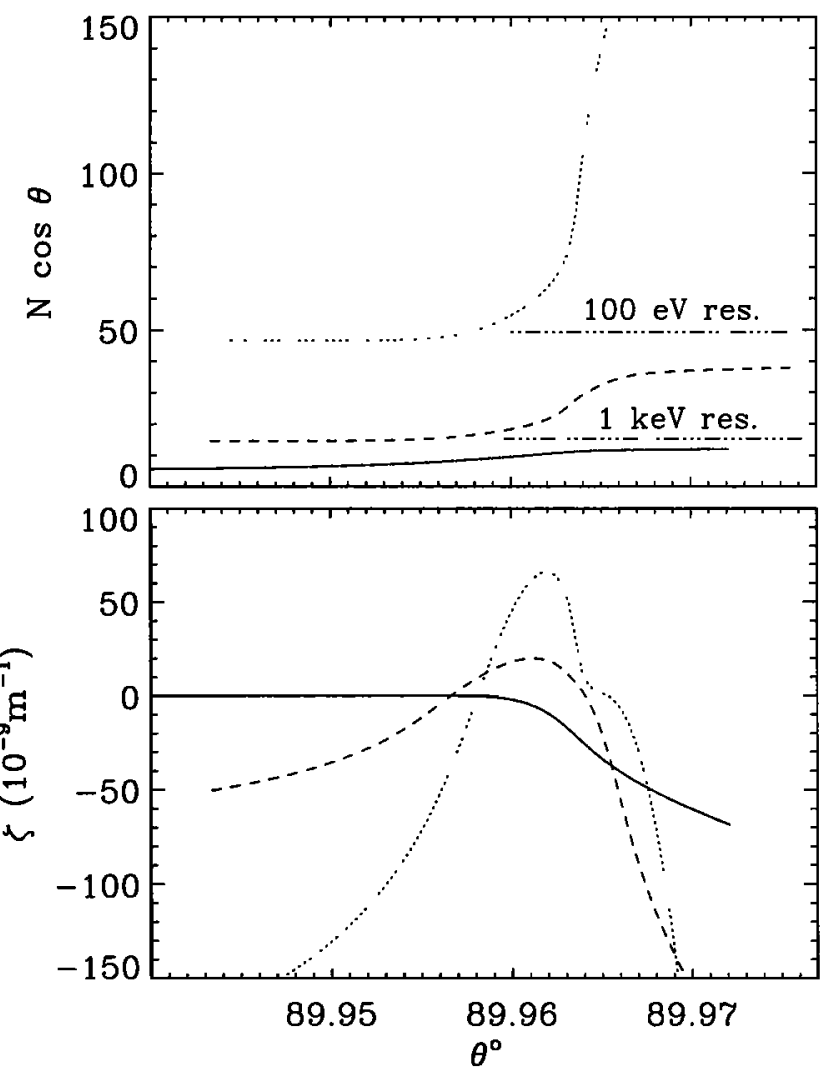

Figure 9. Parallel projection of the (top) refractive index and the (bottom) spatial growth rate $\zeta$, plotted versus $\theta$. The plasma model is assumed to be as in Figure 8, with a frequency corresponding to the higher-frequency peak in the measured spectrum (130 $\left.\mathrm{Hz} \approx \frac{1}{3} \Omega_{\mathrm{H}} / 2 \pi\right)$. The solid line corresponds to zero ion temperature (cold-plasma approximation), the dashed line corresponds to Maxwellian ions with a temperature of $1 \mathrm{eV}$, and the dotted line represents the ion temperature of $10 \mathrm{eV}$. The resonance condition for the $1-\mathrm{keV}$ electron beam corresponds to the horizontal line in $\mathrm{Fi}$ gure 9 (top), where the beam velocity $\left(v_{b} \approx 1.88 \times 10^{8}\right.$ $\mathrm{m} / \mathrm{s}$ ) is equal to phase velocity $v_{f}$. 
The influence of a finite ion temperature is shown in Figure 9. The solid lines present a horizontal cut of Figure 8 for the frequency $130 \mathrm{~Hz}$ (the location of the higher frequency peak in the measured spectrum). The dashed and dotted lines were calculated using the same plasma model, except for the temperature of ion components. The cold-plasma approximation has been replaced by a Maxwellian distribution with the temperatures $1 \mathrm{eV}$ (dashed line) and $10 \mathrm{eV}$ (dotted line). The approximate condition for the Landau resonance (the phase-velocity projection equal to the beam velocity) is plotted in Figure 9 (top). Near the $\theta$ value, where the condition is fulfilled, the positive peak of the wave growth occurs. Its shape is almost independent of the ion temperature. For a higher $\theta$ a flat secondary peak is observed. Its width is strongly dependent on the ion temperature. This interval of weakly growing waves may be interpreted as a resonance on the proton gyrofrequency. As demonstrated, the finite ion temperature has no substantial influence on the total wave growth, because the Landau resonance is the main generation mechanism at observed frequencies.

We also analyzed the wave stability in the observed low-frequency band of EMIC waves. The results, similar to those presented in Figure 8, were obtained for the same plasma model. The general shape of the dependence of wave growth on $\theta$ and frequency has been conserved. The only differences are a more compressed scale of $\theta$ values (the hot-plasma effects are observed in a scale of $0.01^{\circ}$ ) and much lower absolute values of the spatial growth rate. At $8 \mathrm{~Hz}$ (the maximum intensity in the lower band of the measured spectrum), wave growth reaches only about $6 \%$ of the value at $130 \mathrm{~Hz}$.

To study how the wave growth depends on the relative abundance of beam electrons and on the drift energy of the beam, the original plasma model from Figure 7 has been assumed. Two dense electron beams with energies of $100 \mathrm{eV}$ and $1 \mathrm{keV}$ are expected to increase substantially the wave growth at $8 \mathrm{~Hz}$. Figure 10 presents the obtained results in a form similar to that in Figure 9. Near the resonance angle, the solution of the dispersion relation is split into three independent branches. The conditions for the Landau resonance of both beams are plotted in Figure 10 (top), which shows that two branches contain the growing waves (dashed and dotted lines). Each of them fulfills the resonance condition of the respective beam. The third branch (solid line) follows the cold-plasma solution and becomes damped near the cold-plasma resonance. The influence of the respective plasma components is completely separated. If just one beam is present, only the corresponding branch exists without substantial changes. In accordance with the results of Temerin and Lysak [1984], the 100-eV beam causes greater spatial wave growth than the $1-\mathrm{keV}$ beam. However, the temporal growth $\Im \omega / \Re \omega$ is approximately equal for both beams, and the difference is due to a lower group velocity of the $100-\mathrm{eV}$ branch. A flat secondary peak on the $100-\mathrm{eV}$ branch is interpreted as the resonance on the first harmonic of the oxygen gyrofrequency $(25 \mathrm{~Hz})$. Its

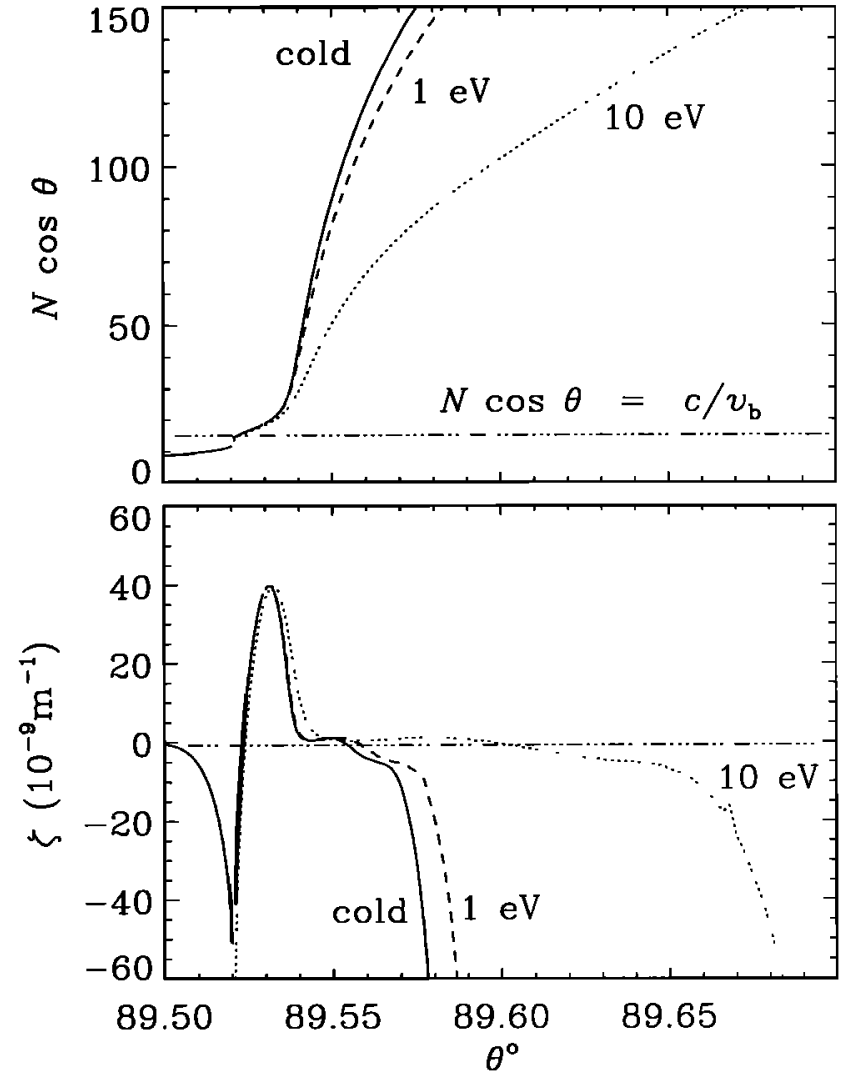

Figure 10. Same as Figure 9, but with the plasma model with two dense electron beams as in Figure 7 used to study the increase of wave growth in the lowfrequency band $\left(8 \mathrm{~Hz} \approx \frac{1}{3} \Omega_{\mathrm{O}^{+}} / 2 \pi\right)$ of observed EMIC waves. Three separate branches are marked with different line styles. The solid line corresponds to a damped branch connected with the Maxwellian plasma, the dashed line represents a branch resonant with the 1-keV beam, and the dotted line is a branch resonant with the $100-\mathrm{eV}$ beam. The resonance condition for both electron beams is drawn.

intensity is again much lower than the growth due to the Landau resonance.

With the plasma model containing more free energy, same-order values of the wave growth have been obtained as for the previous results in the higher-frequency band. However, with the same model the wave dispersion at $130 \mathrm{~Hz}$ exhibits similar properties as reported in Figure 10, and the wave growth is again more than 16 times higher than at $8 \mathrm{~Hz}$. Note that the influence of increasing the beam density is not only quantitative; the behavior of wave dispersion near the resonance qualitatively changes and new EMIC branches occur. The effect of beam temperature has been studied without obtaining new information about the rate of wave growth in the higher and lower EMIC bands. While decreasing the thermal spread of the beam, the growth rate increases in both bands by approximately the same factor. The results of the presented preliminary analysis of linear instability of EMIC waves disagree with the observed spectrum, mainly in the following points. (1) 
The wave growth obtained for the low-frequency band is only about $6 \%$ of the growth in the higher-frequency band, but the waves are observed in both bands. (2) In both bands a higher growth has been obtained for higher frequencies. The slope is rather inverse in the measured spectrum.

Some effects probably cannot be explained by the linear theory of homogeneous media that was used in our analysis. The disagreement may be also due to a difference of the assumed plasma model from the conditions in the generation region, in which case, propagation effects should be taken into account. The reconstruction of the WDF can be an important tool in the analysis of EMIC waves. Propagation effects may lead to splitting of wave normals into two different directions [Parrot and Lefeuvre, 1986], or the observed wave normals can be declined from their original directions. The WDF reconstruction on selected frequencies would therefore help to find the most probable interpretation of the observed spectrum.

\subsection{Influence of the Doppler Effect}

The Doppler effect was not taken into account in any of the cases presented above. If a satellite velocity of 5 $\mathrm{km} / \mathrm{s}$ is supposed, a nonnegligible bias of the observed frequency requires a relatively high refractive index of several thousands. In the case of Figure 9 or 10 this condition is fulfilled and the Doppler effect should be taken into account. To do this, we need information about the wave normal directions of the observed waves. The reconstruction of the WDF or an equivalent approach is therefore necessary. Figure 11 presents an example of effects which can be obtained when the Doppler effect is taken into account. A plasma model as in the case of cold ions in Figure 9 is supposed. The solid line represents the same solution of the dispersion relation as presented by the solid line in Figure 9; that is, the satellite velocity $\mathbf{v}_{O}$ is supposed to be perpendicular to the wave normal, and no Doppler effect is seen.

The dashed line corresponds to the satellite velocity nearly antiparallel to the wave normal. The component $v_{O 1(p)}$ of the velocity (i.e., the projection of $\mathbf{v}_{O}$ to the axis $x_{1(p)}$ of the coordinate system of Figure 1$)$ is equal to $5 \mathrm{~km} / \mathrm{s}$ and the wave normal is defined by the angle $\phi=180^{\circ}$. For lower $\theta$ values the refractive index is not sufficiently high to observe an influence of the Doppler effect. If $\theta>89.55^{\circ}$, the Doppler effect causes decreasing of the refractive index and an extension of the interval where the hot plasma effects are observed.

The dotted line presents the results when the satellite velocity is nearly parallel to the wave normal. The normal is now defined by $\phi=0^{\circ}$ for the same component $v_{01(p)}$ of the velocity. For lower $\theta$ values and lower refractive index, the results are again identical to those in the case without the Doppler effect. However, as the refractive index increases, important differences are obtained. At the $\theta$ value, where the maximum wave amplification is observed, the EMIC mode solution is mirrored to the lower $\theta$ values, with increasing refractive index. This phenomenon has a clear consequence con-

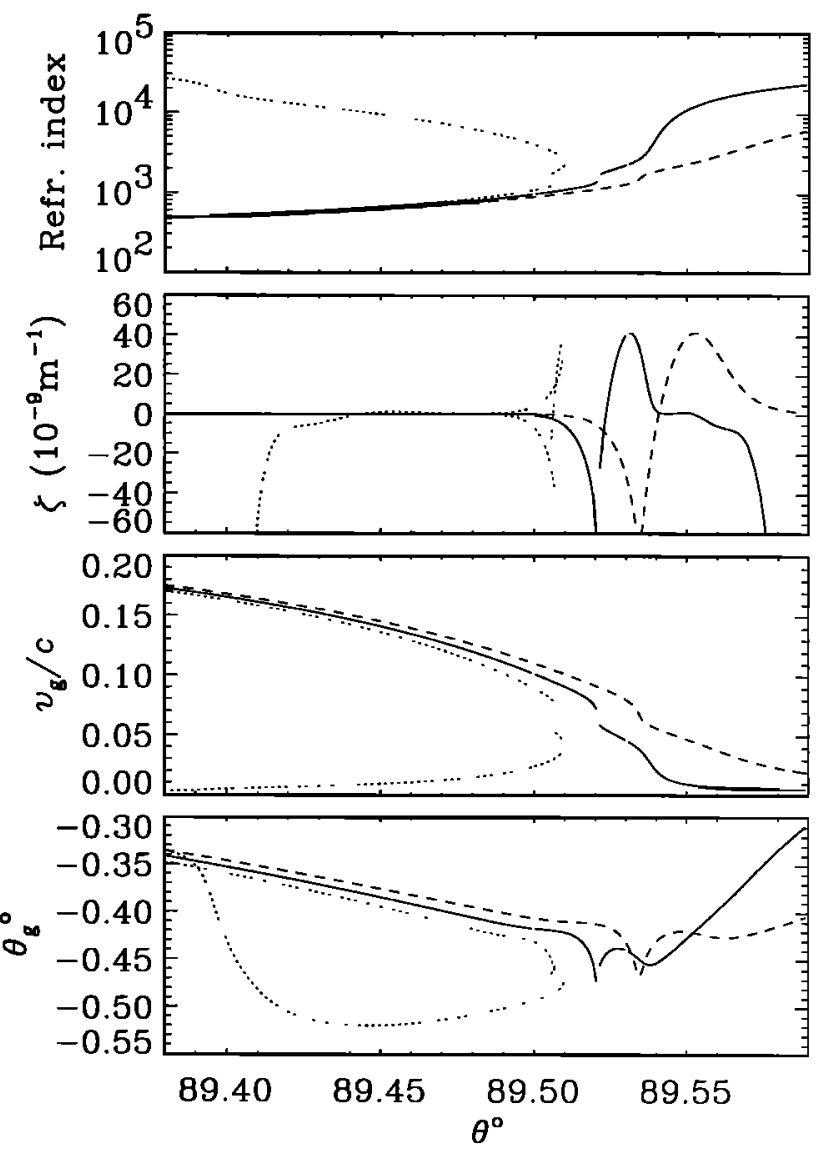

Figure 11. Dependence of the solution of the Dopplershifted dispersion relation on $\theta$. (top to bottom) refractive index, spatial growth rate, group-velocity modulus normalized to the velocity of light, and deviation of the group velocity from the ambient magnetic field. The solid line corresponds to the case without the Doppler effect, the solution taking into account the velocity $v_{O 1(p)}$ of $5 \mathrm{~km} / \mathrm{s}$ is presented by the dotted line, and the dashed line corresponds to an opposite velocity. The plasma model is assumed as in Figure 8, with a frequency corresponding to the higher-frequency peak in the measured spectrum $\left(130 \mathrm{~Hz} \approx \frac{1}{3} \Omega_{\mathrm{H}^{+}} / 2 \pi\right)$.

cerning the WDF problem; the multimodal approach described in section 2 must be used.

\section{Conclusions}

This paper contains a detailed characterization of the WDF in a hot plasma. A resolution of the direct problem has been made, taking into account the Doppler effect. Our approach is essentially similar to the coldplasma method of Storey and Lefeuvre [1980], and it is different from Oscarsson and Rönnmark [1989]. We use an explicit frequency dependence of the WDF which is well related to the experimental data recorded by satellites.

Our detailed theoretical analysis of different waves in a hot plasma has provided a description of physical quantities (wave refractive index, wave growth rate, 
group velocity, electromagnetic field intensities), which have been computed with plasma parameters that are relevant to different experimental observations. The difference between cold- and hot-plasma solution is shown. Important effects are mainly observed around the resonance angle. Waves propagating with high values of refractive index are strongly influenced by the Doppler effect. The detailed calculations have demonstrated that the Doppler effect may increase the number of wave branches which can be observed at a given frequency and for a fixed wave-normal direction.

Further work needs to be done now to solve the inverse problem of the WDF, i.e., to find the shape of the WDF using experimental data. The problem is more complicated than that for the cold-plasma approximation, because the solution of the dispersion relation indicates that several wave modes are often observed for a given $\theta$ angle.

Acknowledgments. The work of $O$. Santolík was supported by the Ministère de l'Enseignement Supérieur et de la Recherche (France). He also acknowledges the support of the Czech Grant Agency grant 202/94/0467.

The Editor thanks referee 1 and $M$. Hayakawa and $S$. Sazhin for their assistance in evaluating this paper.

\section{References}

Arthur, C. W., R.L. McPherron, and J.D. Means, A comparative study of three techniques for using the spectral matrix in the wave analysis, Radio Sci., 11, 833-845, 1976.

Cornilleau-Wehrlin, N., et al., The STAFF experiment in Cluster: Mission payload and supporting activities, Eur. Space Agency Spec. Publ., ESA SP-1159, 33-48, 1993.

Delannoy, C., and F. Lefeuvre, MAXENTWDF - A computer program for the maximum entropy estimation of a wave distribution function, Comp. Phys. Commun., 40, 389-419, 1986.

Erlandson, R. E., L. J. Zanetti, M. H. Acuña, A. I. Eriksson, L. Eliasson, M. H. Boehm, and L. G. Blomberg, Freja observations of electromagnetic ion cyclotron ELF waves and transverse oxygen ion acceleration on auroral field lines, Geophys. Res. Lett., 21, 1855-1858, 1994.

Gustafsson, G., M. André, L. Matson, and H. Koskinen, On waves below the local proton gyrofrequency in auroral acceleration regions, J. Geophys. Res., 95, 5889-5904, 1990.

Horne, R. B., and S.S. Sazhin, Quasielectrostatic and electrostatic approximations for whistler mode waves in the magnetospheric plasma, Planet. Space Sci., 38, 311-318, 1990.

Lefeuvre, F., Analyse de champs d'ondes électromagnétiques aléatoires observées dans la magnétosphère, à partir de la mesure simultanée de leurs six composantes, Doctoral thesis, Univ. of Orléans, Orléans, France, 1977.

Lefeuvre, F., and C. Delannoy, Analysis of random electromagnetic wave field by a maximum entropy method, $A n n$. Telecommun., 34, 204-213, 1979.

Lefeuvre, F., J. L. Rauch, D. Lagged, J. J. Berthelier, and J. C. Cerisier, Propagation characteristics of dayside lowaltitude hiss: Case studies, J. Geophys. Res., 97, 10,60110,620, 1992 .

McPherron, R. L., C. T. Russel, and P. J. Coleman Jr., Fluctuating magnetic fields in the magnetosphere, 2, ULF waves, Space Sci. Rev., 13, 411-454, 1972.
Means, J. D., Use of the three-dimensional covariance matrix in analyzing the polarization properties of plane waves, J. Geophys. Res., 77, 5551-5559, 1972.

Oscarsson, T., Stability analysis and reconstruction of wave distribution functions in warm plasmas, Ph.D. thesis, Univ. of Umeå, Umeå, Sweden, 1989.

Oscarsson, $T$., Dual principles in maximum entropy reconstruction of the wave distribution function, $J$. Comput. Phys., 110, 221-233, 1994.

Oscarsson, T., and K. Rönnmark, Reconstruction of wave distribution functions in warm plasmas, J. Geophys. Res., 94, 2417-2428, 1989

Oscarsson, T., and K. Rönnmark, A combined wave distribution function and stability analysis of Viking particle and low-frequency data, J. Geophys. Res., 95, 21,18721,202, 1990.

Parrot, M., and F. Lefeuvre, Statistical study of the propagation characteristics of ELF hiss observed on GEOS-1, outside and inside the plasmasphere, Ann. Geophys., 4, 363-384, 1986.

Rönnmark, K., Computation of the dielectric tensor of a Maxwellian plasma, Plasma Phys., 25, 699-701, 1983.

Rönnmark, K., and J. Larsson, Local spectra and wave distribution function, J. Geophys. Res., 93, 1809-1815, 1988.

Samson, J. C., and J. V. Olson, Some comments on the descriptions of the polarisation states of waves, Geophys. J. R. Astron. Soc., 61, 115-129, 1980.

Sazhin, S.S., An approximate theory of electromagnetic wave propagation in a weakly relativistic plasma, $J$. Plasma Phys., 37, 209-230, 1987.

Sazhin, S.S., Oblique whistler-mode growth and damping in a hot anisotropic plasma, Planet. Space Sci., 36, 663-667, 1988.

Sazhin, S.S., Whistler-Mode Waves in a Hot Plasma, Cambridge Univ. Press, New York, 1993.

Sazhin, S.S., and R. B. Horne, Quasilongitudinal approximation for whistler-mode waves in the magnetospheric plasma, Planet. Space Sci., 38, 1551-1553, 1990.

Stix, T. H., The Theory of Plasma Waves, McGraw-Hill, New York, 1962.

Storey, L. R. O., and F. Lefeuvre, Theory for the interpretation of measurements of a random electromagnetic wave field in space, Space Res., 14, 381-386, 1974.

Storey, L. R. O., and F. Lefeuvre, The analysis of 6component measurement of a random electromagnetic wave field in a magnetoplasma, 1, The direct problem, Geophys. J. R. Astron. Soc., 56, 255-270, 1979.

Storey, L. R. O., and F. Lefeuvre, The analysis of 6component measurement of a random electromagnetic wave field in a magnetoplasma, 2, The integration kernels, Geophys. J. R. Astron. Soc., 62, 173-194, 1980

Temerin, M., and R. L. Lysak, Electromagnetic ion cyclotron mode (ELF) waves generated by auroral electron precipitation, J. Geophys. Res., 89, 2849-2859, 1984.

M. Parrot, Laboratoire de Physique et Chimie de l'Environnement, CNRS, 3A, Avenue de la Recherche Scientifique, 45071 Orléans cedex 02, France. (e-mail: mparrot@cnrs-orl zans.fr)

O. Santolík, Faculty of Mathematics and Physics, Charles University, V Holešovičkách 2, CZ-18000 Praha 8, Czech Republic. (e-mail: santolik@hp03.troja.mft.cuni.cz)

(Received March 7, 1995; revised November 2, 1995; accepted November 7,1995 .) 